\title{
Adult autism spectrum as a transnosographic dimension
}

\author{
Liliana Dell'Osso, ${ }^{\prime *}$ Riccardo Dalle Luche, ${ }^{\prime}$ and Mario $\mathrm{Maj}^{2}$
}

\author{
${ }^{1}$ Department of Clinical and Experimental Medicine, Psychiatry Sector, University of Pisa, Pisa, Italy \\ ${ }^{2}$ Department of Psychiatry, University of Naples SUN, Naples, Italy
}

Received 9 February 2015; Accepted 25 March 2015; First published online 9 September 2015

Autism is usually diagnosed in childhood on the basis of early impairment in reciprocal communication and social interaction, stereotypic behaviors, late or poor language development, or intellectual retardation. However, subjects with mild forms of autism, with normal or aboveaverage intelligence, may reach high levels of functioning, developing in some cases excellent abilities in restricted areas, and may be undiagnosed until adulthood or misdiagnosed as having other mental disorders. Growing interest has been devoted in the last several years to the adult courses of these conditions, previously labelled as Asperger's disorder $(\mathrm{AD})$ and recently merged into the autism spectrum disorder (ASD) in the Fifth Edition of the Diagnostic and Statistical Manual of Mental Disorders (DSM-5), along with growing literature indicating that they are associated with high rates of comorbidity with anxiety, mood, psychotic, and trauma- and stress-related disorders and with suicidal behaviors. ${ }^{1-3}$

While DSM-5 made the attempt to fill the gaps between diagnostic categories by applying a spectrum approach to neurodevelopmental disorders, ASD diagnostic criteria may still be not sensitive enough to detect some adult forms with no intellectual or language disability and characterized by mild impairment in interpersonal and empathic functions, such as attuning and socialization, leading to isolation and social phobias. Individuals with such forms, lying at the boundaries of ASD, might be easily misdiagnosed as having a wide range of personality disorders (eg, schizoid, schizotypal, avoidant, dependent, borderline) or unspecified schizophrenia spectrum disorders and other psychotic disorders.

Looking back at Asperger's original descriptions, he focused on some stable autistic traits, often associated with special abilities, referring to Eugen Bleuler's concept of autism as a fundamental symptom of

*Address for correspondence: Liliana Dell'Osso, University of Pisa Department of Clinical and Experimental Medicine, Psychiatry Unit, via Roma 67, 56100 Pisa, Italy.

(Email: liliana.dellosso@med.unipi.it) schizophrenia and somehow recalling Jung"s "introvert" psychological type and Kretschmer's "schizoid psychopathy." Since entering the DSM in 1994, AD has lost the distinctiveness of Asperger's first observations. Moreover, diagnostic criteria have mostly referred to the childhood course rather than to the adult clinical phenotype. From this perspective, there is no blame for dropping $\mathrm{AD}$ as a separate category in DSM-5. However, Asperger's work should be revised within a coherent adult autism spectrum model that is currently supported by both clinical and neurobiological data.

Consistent with Asperger's intuition, it has been proposed that a neurodevelopmental deviation may represent the common vulnerability factor for most mental disorders (eg, schizophrenia, bipolar disorder, attention deficit hyperactivity disorder), as suggested by their clinical overlap, familial co-aggregation, and shared genetic risk factors with ASD. Along with this hypothesis, genetic and environmental factors would act together to cause a neurodevelopmental deviation, the timing, severity, and anatomical location of which determine a gradient of neuroatypicality underlying different mental conditions. ${ }^{4}$ Somehow consistent with this hypothesis, it has been proposed that mental disorders should be reformulated as pathologies of the whole-brain organization, or "globalopathies," rather than disturbances of a discrete neural network. ${ }^{5,6}$ If a global, brain-based physiopathology must be hypothesized for psychiatric conditions, ${ }^{7}$ this might involve neurodevelopmental processes and the extent to which the brain is neuroatypic. This idea is also in line with some new and intriguing perspectives derived from neurobiological studies that have raised the hypothesis that schizophrenia could be considered a late-onset neurodevelopmental disorder, ${ }^{8}$ or the late stage of one, ${ }^{9}$ rather than an earlyonset neurodegenerative disease, as initially suggested by the Kraepelinian definition of dementia praecox.

Interestingly, growing bodies of data indicate that ASD often remains unrecognized until adulthood, coming to clinical attention only when other mental disorders arise, 
often prompted by a breakdown of relations, such as a parent's death or other life events and changes, leading to challenging diagnostic procedures. ${ }^{10}$ This is likely the case of ASD patients with comorbid complex posttraumatic stress disorder (PTSD) who may be diagnosed as borderline personality disorder, while the ASD goes unrecognized. ${ }^{11}$ The same idea applies to those ASD adults with comorbid mood disorders who are often diagnosed as schizophrenics because of the atypical mood episodes and inter-episodic behavioral patterns related to ASD. ${ }^{12}$ Moreover, ASD seems to contribute to impulsive suicidal behaviors in the context of many different comorbid mental disorders. ${ }^{2,13,14}$

We suggest an adult autism spectrum (AdAS) model that would encompass not only threshold-level symptoms but also mild and atypical manifestations, behavioral traits, and personality features associated with the DSM-5 ASD diagnostic category. These may, or may not, interfere with overall functioning and quality of life, ${ }^{10}$ ending in the continuum of normality and even including positive aspects of neuroatypicality and some hyper-adaptive features such as originality, creativity, divergent thinking, ${ }^{15,16}$ thus overcoming the distinction between symptom and trait.

From a clinical standpoint, we hypothesize that the use of the AdAS model will help both to detect high-risk subjects before they develop other mental disorders, and to identify ASD-colored clinical presentations in the context of these latter, ${ }^{11}$ when a mild/subthreshold autism spectrum is present. More speculatively, the AdAS will also help to investigate whether, besides its role as a vulnerability factor for other mental disorders, neuroatypicality may also increase patients' resilience to certain kinds of life events.

From the point of view of research, the AdAS approach will help to investigate the genetic basis of autism. The DSM-5, in fact, still draws a line between what is autism and what is not, ${ }^{17}$ disregarding more subtle phenomena such as the broad autism phenotype or the subthreshold autism spectrum, ${ }^{18}$ spanning across the general population who do not fully meet criteria for $\mathrm{ASD}^{19}$ but who share, however, genetic underpinnings with the clinical population. ${ }^{20-23}$ While a reliable threshold for the diagnosis is essential in clinical practice, our search for neurobiological mechanisms may need some intermediate entities between the presence and the absence of a mental disorder. ${ }^{24}$ From this perspective, the AdAS approach will allow us to investigate more comprehensively the genetic underpinnings of autism, providing researchers with a broader range of autistic endophenotypes to be tested in their studies. $^{25,26}$

Further, such a spectrum approach has the potential to overcome the descriptive artifice of comorbidity. ${ }^{27}$ According to Krueger and Markon, ${ }^{28}$ who proposed a "liability spectrum model" of comorbidity, mental disorders are the manifestation of latent liability factors that explain comorbidity by virtue of their impact on multiple disorders. ${ }^{29}$ According to such a model of comorbidity, the AdAS could help to assess individual deviations along a number of autistic dimensions, which may predispose patients to several mental disorders, that would explain the high rates of comorbidity between ASD and other psychiatric conditions. Just as a panic attack is conceptualized not only in the context of a specific disorder, but also as a transnosographic specifier for different disorders in the DSM-5, in the same way the assessment of AdAS could help clinicians to better define the physiopathology, course, vulnerability/resilience factors, and specificity in pharmacological and psychological treatment response of most mental disorders. From this perspective, some dimensions underlying autistic symptomatology (eg, social phobias, social withdrawal, proneness to rumination, obsessive perfectionism, rigidity, clumsiness, disturbance of empathy, anhedonia) could represent a sort of common autistic core of several mental conditions. $^{30-32}$

Ruminative thoughts, for example, have been shown to be associated with PTSD ${ }^{33-35}$ and the literature indicates a high incidence of multiple traumas in individuals with ASD, such that a few authors have speculated that ASD constitutes a vulnerability factor to PTSD after trauma exposure. ${ }^{11}$ Taken together, these data may suggest the intriguing hypothesis that subjects with ASD might be at higher risk of developing trauma- and stress-related disorders due to their proneness to rumination. We think that the revision and reconceptualization of Asperger's work within the framework of such an AdAS model will allow the detection of symptoms that clinicians usually overlook, but which interfere with the vulnerability and the clinical presentation of most mental disorders. Eventually, we argue the AdAS model as a transnosographic dimension based on a neurodevelopmental physiopathology of mental disorders.

\section{Disclosures}

The authors do not have an affiliation with or financial interest in any organization that might pose a conflict of interest.

\section{REFERENCES:}

1. Kato K, Mikami K, Akama F, et al. Clinical features of suicide attempts in adults with autism spectrum disorders. Gen Hosp Psychiatry. 2013; 35(1): 50-53.

2. Storch EA, Sulkowski ML, Nadeau J, et al. The phenomenology and clinical correlates of suicidal thoughts and behaviors in youth with autism spectrum disorders. J Autism Dev Disord. 2013; 43(10): 2450-2459.

3. Vannucchi G, Masi G, Toni C, Dell’Osso L, Marazziti D, Perugi G. Clinical features, developmental course, and psychiatric 
comorbidity of adult autism spectrum disorders. CNS Spectr. 2014; 19(2): 157-164.

4. Doherty JL, Owen MJ. Genomic insights into the overlap between psychiatric disorders: implications for research and clinical practice. Genome Med. 2014; 6(4): 29.

5. Peled A, Geva AB. "Clinical brain profiling": a neuroscientific diagnostic approach for mental disorders. Med Hypotheses. 2014; 83(4): 450-464.

6. Peled A. Brain "globalopathies" cause mental disorders. Med Hypotheses. 2013; 81(6): 1046-1055.

7. White PD, Rickards H, Zeman AZ. Time to end the distinction between mental and neurological illnesses. BMJ. 2012; 344: e3454.

8. Paus T, Keshavan M, Giedd JN. Why do many psychiatric disorders emerge during adolescence? Nat Rev Neurosci. 2008; 9(12): 947-957.

9. Insel TR. Rethinking schizophrenia. Nature. 2010; 468(7321): 187-193.

10. Kamio Y, Moriwaki A, Takei R, et al. [Psychiatric issues of children and adults with autism spectrum disorders who remain undiagnosed]. Seishin Shinkeigaku Zasshi. 2013; 115(6): 601-606.

11. King R. Complex post-traumatic stress disorder: implications for individuals with autism spectrum disorders-part 1. Journal on Developmental Disabilities. 2010; 16(3): 91-100.

12. Skeppar P, Adolfsson R. Bipolar II and the bipolar spectrum. Nord J Psychiatry. 2006; 60(1): 7-26.

13. Simoncini M, Miniati M, Vanelli F, et al. Lifetime autism spectrum features in a patient with a psychotic mixed episode who attempted suicide. Case Rep Psychiatry. 2014; 2014: 459524.

14. Takara K, Kondo T. Comorbid atypical autistic traits as a potential risk factor for suicide attempts among adult depressed patients: a case-control study. Ann Gen Psychiatry. 2014; 13(1): 33.

15. Baron-Cohen S, Richler J, Bisarya D, Gurunathan N, Wheelwright S The systemizing quotient: an investigation of adults with Asperger syndrome or high-functioning autism, and normal sex differences. Philos Trans R Soc Lond B Biol Sci. 2003; 358(1430): 361-374.

16. Tantam D, Girgis S. Recognition and treatment of Asperger syndrome in the community. Br Med Bull. 2009; 89(1): 41-62.

17. Happé F. Criteria, categories, and continua: autism and related disorders in DSM-5. J Am Acad Child Adolesc Psychiatry. 2011; 50 (6): $540-542$.

18. Nemeroff CB, Weinberger D, Rutter M, et al. DSM-5: a collection of psychiatrist views on the changes, controversies, and future directions. BMC Med. 2013; 11: 202.

19. Scheeren AM, Stauder JE. Broader autism phenotype in parents of autistic children: reality or myth? J Autism Dev Disord. 2008; 38(2): 276-287.

20. Le Couteur A, Bailey A, Goode S, et al. A broader phenotype of autism: the clinical spectrum in twins. J Child Psychol Psychiatry. 1996; 37(7): 785-801.

21. Smith CJ, Lang CM, Kryzak L, Reichenberg A, Hollander E, Silverman JM. Familial associations of intense preoccupations, an empirical factor of the restricted, repetitive behaviors and interests domain of autism. J Child Psychol Psychiatry. 2009; 50(8): 982-990.

22. Constantino JN, Zhang Y, Frazier T, Abbacchi AM, Law P. Sibling recurrence and the genetic epidemiology of autism. Am J Psychiatry. 2010; 167(11): 1349-1356.

23. Ronald A, Hoekstra RA. Autism spectrum disorders and autistic traits: a decade of new twin studies. Am J Med Genet B Neuropsychiatr Genet. 2011; 156B(3): 255-274.

24. Maj M. DSM-5, ICD-11 and 'pathologization of normal conditions'. Aust N Z J Psychiatry. 2014; 48(2): 193-194.

25. Gottesman II, Gould TD. The endophenotype concept in psychiatry: etymology and strategic intentions. Am J Psychiatry. 2003; 160(4): 636-645.

26. Sucksmith E, Roth I, Hoekstra RA. Autistic traits below the clinical threshold: re-examining the broader autism phenotype in the 21st century. Neuropsychol Rev. 2011; 21(4): 360-389.

27. Maj M. "Psychiatric comorbidity": an artefact of current diagnostic systems? Br J Psychiatry. 2005; 186(3): 182-184.

28. Krueger RF, Markon KE. Reinterpreting comorbidity: a modelbased approach to understanding and classifying psychopathology. Annu Rev Clin Psychol. 2006; 2: 111-133.

29. Dell'Osso L, Pini S. What did we learn from research on comorbidity in psychiatry? Advantages and limitations in the forthcoming DSM-V era. Clin Pract Epidemiol Ment Health. 2012; 8: 180-184.

30. Chanachev A, Berney A. Apathy, a transnosographic symptom: diagnosis and treatment. Rev Med Suisse. 2010; 6(236): 326-329.

31. Chevallier C, Grèzes J, Molesworth C, Berthoz S, Happé F. Brief report: Selective social anhedonia in high functioning autism. J Autism Dev Disord. 2012; 42(7): 1504-1509.

32. Del-Monte J, Capdevielle D, Gély-Nargeot MC, et al. [Evolution of the concept of apathy: the need for a multifactorial approach in schizophrenia]. Encephale. 2013; 39(Suppl 1): S57-S63.

33. Ben-Sasson A, Cermak SA, Orsmond GI, Tager-Flusberg H, Kadlec MB, Carter AS. Sensory clusters of toddlers with autism spectrum disorders: differences in affective symptoms. J Child Psychol Psychiatry. 2008; 49(8): 817-825.

34. Lecavalier L, Gadow KD, Devincent CJ, Houts CR, Edwards MC. Validity of DSM-IV syndromes in preschoolers with autism spectrum disorders. Autism. 2011; 15(5): 527-543.

35. Foley Nicpon M, Doobay AF, Assouline SG. Parent, teacher, and self perceptions of psychosocial functioning in intellectually gifted children and adolescents with autism spectrum disorder. J Autism Dev Disord. 2010; 40(8): 1028-1038. 